\title{
Evaluation of Inter-professional Education (IPE) with medical, nursing and pharmacy students through a simulated IPL Educational Intervention
}

\author{
Peter Hamilton ${ }^{1}$, Claire Coey-Niebel ${ }^{2}$, Jennifer McCaig ${ }^{3}$, Leon Zlotos ${ }^{1}$, Ailsa Power ${ }^{1}$, Gail \\ Craig $^{1}$, Sheelagh Peacock ${ }^{4}$, and Catherine Paton ${ }^{4}$ \\ ${ }^{1}$ NHS Education for Scotland \\ ${ }^{2}$ NHS Greater Glasgow and Clyde \\ ${ }^{3}$ NHS Ayrshire and Arran \\ ${ }^{4}$ NHS Lanarkshire
}

March 31, 2021

\begin{abstract}
Introduction Inter-professional education (IPE) is becoming an integral part of many professional programmes throughout the UK, ensuring health professionals are competent to work as part of an inter-professional team upon entry into their profession. IPE has become a fundamental component of curriculum content in health and social care degrees. Aims Research aim - to evaluate a simulated IPE intervention. Methods A one day IPE intervention, 'Evening On-Call' was run involving nursing and medical students and pre-registration pharmacists (student pharmacists in year 5 of training) in an on-call setting. This IPE incorporated manikin and actor patients in a simulated ward. During the intervention, the 3 groups of students are assessed under observation on their clinical, prioritisation and communication skills. Participants perceptions of this intervention were evaluated by completion of a questionnaire to capture their perceptions regarding the experience, the pre-IPL briefing and post-IPL feedback and perceived relevance of this training. Free text sections collected additional comments and a follow-up questionnaire was sent six months later. Results Initial questionnaire feedback was predominantly positive for each professional group. The majority perceived the simulated IPL had given them a greater understanding of other professionals' roles, had enhanced their professional confidence and would help them prioritise workload once qualified. The 6 months follow up questionnaire supported the initial questionnaire findings. Some responses highlighted participants believed the simulated IPL had helped them work more effectively with other healthcare professionals, communicate more effectively and better prioritise their workload. There may be some evidence of sustained self-reported effectiveness in teaching certain professional and clinical skills to participants using this type of simulated intervention.
\end{abstract}

\section{Hosted file}

Evaluation of a simulated IPL Intervention Manuscript.pdf available at https://authorea. com/users/405097/articles/516119-evaluation-of-inter-professional-education-ipe-withmedical-nursing-and-pharmacy-students-through-a-simulated-ipl-educational-intervention 